\title{
The Immediate Mitochondrial Stress Response in Coping with Systemic Exposure of Silver Nanoparticles in Rat Liver
}

\author{
Tzu-Ying Lee ${ }^{1}$, Maw-Shung Liu ${ }^{2}$, Li-Ju Huang ${ }^{3}$, Sheng-I Lue ${ }^{4}$, Tsen-Ni Tsai ${ }^{1}$, Lung-Chang Lin ${ }^{4}$, Aij-Lie Kwan $^{1,5}$ and Rei-Cheng Yang $^{2,4,6 *}$ \\ ${ }^{1}$ Graduate Institute of Medicine, College of Medicine, Kaohsiung Medical University, Kaohsiung 807, Taiwan \\ ${ }^{2}$ Department of Physiology, School of Medicine, College of Medicine, Kaohsiung Medical University, Kaohsiung 807, Taiwan \\ ${ }^{3}$ Teaching and Research Center, Kaohsiung Municipal Ta-Tung Hospital, Kaohsiung Medical University Hospital, Kaohsiung Medical University, Kaohsiung 807, Taiwan \\ ${ }^{4}$ Department of Pediatrics, Kaohsiung Medical University Hospital, Kaohsiung 807, Taiwan \\ ${ }^{5}$ Department of Neurosurgery, Kaohsiung Medical University Hospital, Kaohsiung 807, Taiwan \\ ${ }^{6}$ Department of Pediatrics, Changhua Christian Hospital, Changhua county 526, Taiwan
}

\begin{abstract}
Silver nanoparticles (Ag-nps) induce hepatotoxicities via oxidative stress. The oxidative stress-induced mitochondrial damages are known to be regulated by mitochondrial unfolded protein response (mtUPR) and autophagy/mitophagy systems. The present study was undertaken to investigate role of mtUPR and autophagy/mitophagy on the Ag-nps induced mitochondrial dysfunction. Experiments consisted of two groups of Sprague Dawley rats: control and Ag-nps treated. The Ag-nps treated group received an intraperitoneally injection of $1.5 \mathrm{ml}$ deionized water containing $500 \mathrm{mg} /$ $\mathrm{kg}$ of Ag-nps. Control group received equal volume of deionized water. All animals were sacrificed at different time points $(6,12,18$ and $24 \mathrm{hr}$ post treatment), followed by removal of livers for determination of ATP content and protein expression. The results show that multi-ubiquitinated proteins (Ub-proteins) in liver mitochondria were increased at $12 \mathrm{hr}$ and the increases were sustained up to $24 \mathrm{hr}$ following Ag-nps treatment. Expressions of mtUPR-associated proteins including transcriptional factors (C/EBP homologous protein (CHOP) and CAAT/enhancer-binding protein- $\beta$ (C/EBP $\beta)$ ), molecular chaperones (heat shock protein (Hsp) 70 and Hsp60) and protease (caseinolytic Clp protease $(\mathrm{ClpP})$ ) remained unchanged during the entire experimental period, except that Hsp10 was upregulated. Expression of LC3-II (autophagy marker) and BNIP3 (mitophagy marker) were upregulated at $6 \mathrm{hr}$ and the upregulation remained throughout the experimental period. ATP content was reduced at $6 \mathrm{hr}$ after Ag-nps exposure and the reductions were sustained during the entire experimental period. These results demonstrate that activation of autophagy/mitophagy markers co-existed with upregulation of Ub-proteins and reduction of ATP content without changing mtUPR in rat liver following Ag-nps administration. These findings indicate that protective effects of autophagy/mitophagy markers were overwhelmed by detrimental actions of Ub-proteins on the control of mitochondrial function, and the counter-balance of the two systems eventually resulting in impaired mitochondrial function, i.e., reduction of ATP content.
\end{abstract}

Keywords: Silver nanoparticles; ATP; Mitochondrial unfolded protein response; Autophagy; Mitophagy

Abbreviations: Ag-nps: Silver Nanoparticles; ATP: Adenosine Triphosphate; mtUPR: Mitochondrial Unfolded Protein Response; Ubproteins: Multi-ubiquitinated Proteins; CHOP: C/EBP Homologous Protein; C/EBP $\beta$ : CAAT/enhancer-binding protein- $\beta$; Hsp70: Heat Shock Protein 70; Hsp60: Heat Shock Protein 60; Hsp10: Heat Shock Protein 10; LC3: Microtubule-associated Protein 1-Light Chain 3; ROS: Reactive Oxygen Species; BNIP3: BCL2/adenovirus E1B 19kDa Protein-interacting Protein 3; PVP: Polyvinylpyrrolidone; VDAC: Voltage-dependent Anion Channel; GAPDH: Glyceraldehyde-3Phosphate Dehydrogenase; i.p: Intraperitoneally; SDS-PAGE: Sodium Dodecyl Sulfate-polyacrylamide Gel Electrophoresis; TBS: Tris-buffer Saline; ECL: Enhanced Chemiluminescence

\section{Introduction}

Unique antibacterial, antiviral and antifungal properties of nanosized silver particles (Ag-nps) render them frequently used in a variety of consumer products ranging from disinfecting medical equipment and water purification systems to textile fabrics and cosmetics [1-4]. For this reason, the toxicity of Ag-nps to human remains an important concern. Several studies indicate that excessive exposure to Ag-nps lead to their accumulation and toxic changes in multiple organs, especially in the liver that functions as a key organ of detoxification [5-7]. Although the mechanisms of hepatotoxicity is not fully understood, prior studies have revealed that the biological and toxic effects of Ag-nps occur due to excessive production of reactive oxygen species (ROS) that lead to eventual apoptosis and necrosis $[1,6,8]$.
ROS are mainly produced in the mitochondria which in turn cause mitochondrial oxidative damage to proteins, membranes, and mitochondrial DNA (mtDNA). The oxidative stress-induced mitochondrial dysfunction occurs when the production of ROS exceeds the antioxidant capacity [9-11]. ROS may modulate proteins through covalent modification of specific reactive cysteine residues and results in misfolded/unfolded protein formations that tend to aggregate and precipitate $[12,13]$. Accumulation of aggregation-prone proteins interferes with normal mitochondrial function, a phenomenon termed mitochondrial proteotoxic stress [14,15]. Apart from ROS production, there are other mechanisms that could explain the toxicity of Ag-nps, including dissolving or releasing of Ag ions from Ag-nps, binding and destruction of proteins or DNA, altering of mitochondrial membrane permeability, and disturbing mitochondrial respiratory chain activities [16-18]. In addition, the toxic mechanisms may involve on the

*Corresponding author: Rei-Cheng Yang, Graduate Institute of Medicine, College of Medicine, Kaohsiung Medical University, Kaohsiung 807, Taiwan, Tel: +886 7 3121101 ext. 2140; Fax: +886 7 3234687; E-mail: rechya@kmu.edu.tw

Received July 14, 2014; Accepted August 20, 2014; Published August 27, 2014

Citation: Lee TY, Liu MS, Huang LJ, Lue SI, Tsai TZN, et al. (2014) The Immediate Mitochondrial Stress Response in Coping with Systemic Exposure of Silver Nanoparticles in Rat Liver. J Nanomed Nanotechnol 5: 220. doi: 10.4172/21577439.1000220

Copyright: (c) 2014 Lee TY, et al. This is an open-access article distributed under the terms of the Creative Commons Attribution License, which permits unrestricted use, distribution, and reproduction in any medium, provided the original author and source are credited. 
properties of the nanoparticles, such as surface area, size and shape, surface charge, particle purity, structural aberration and bioavailability [19].

Several strategies have been developed to monitor mitochondrial damage and ensure the integrity of the cells, including intraorganellar molecular chaperones and proteases that have capacity in regulating and maintaining respiratory chain activity. In response to mitochondrial stress, cells have evolved an auto-regulatory response as the first line of defense at the molecular level, termed mitochondrial unfolded protein response (mtUPR). The mtUPR activates transcriptionally the nuclear-encoded mitochondrial chaperones and proteases to ensure mitochondrial protein quality control [20-23]. Overexpressions of aggregate-forming proteins in the mitochondrial matrix results in an increased expression of mitochondrial molecular chaperones including heat shock protein 70 (Hsp70), heat shock protein 60 (Hsp60), heat shock protein 10 (Hsp10), mitochondrial isoform of DnaJ ( $\mathrm{mtDnaJ}$ ) and the caseinolytic Clp protease (ClpP). Overexpression of these molecular chaperones and proteases facilitate the misfolded protein refolding to normal conformation or selectively remove damaged proteins $[15,22$ 24]. Upregulation of these genes during mtUPR activation was the result of dimerization of CHOP (C/EBP homologous protein) and $\mathrm{C} /$ EBP $\beta$ (CAAT/enhancer-binding protein- $\beta$ ), that in turn promotes the transcription of the mtUPR-responsive genes containing the $\mathrm{CHOP}$ element $[14,22,23]$, and consequently upregulating expression of mitochondrial chaperones and proteases.

In addition to mtUPR, another mitochondrial communication process such as mitophagy, acts as a second line of defence at the organellar level by removing malfunctioning mitochondria through a highly selective form of autophagy $[20,25,26]$. Recent studies have demonstrated that autophagy receptors on damaged mitochondria such as BCL2/adenovirus E1B 19-kDa protein-interacting protein 3 (BNIP3) promote mitophagy via binding of LC3 on autophagosome, followed by delivering BNIP3-LC3-autophagosome complexes to lysosomes for digestion [27,28]. Moreover, mitophagy may also be important in attenuating apoptosis or necrosis through clearance of damaged mitochondria, that in turn prevent production of excess ROS and interrupt the release of cytochrome c, apoptosis-inducing factor, and other apoptotic factors, from intermembrane space [29]. These reports demonstrate that mitophagy plays an important role on the degradation of damaged mitochondria. It should be noted that dysfunctional mitochondria is linked to multiple human pathologies, including obesity, diabetes, cancer, aging, and a number of neurodegenerative and muscular diseases. Factors that promote mitophagy may include growth factor deprivation, TNF- $a$ upregulation, starvation, rapamycin, staurosporine and other toxins [30].

Since mtUPR and mitophagy are two important mechanisms that regulate and control mitochondrial integrity as described in the preceding paragraph, and since mitochondrial integrity, i.e., energy production, was found to be perturbed as early as day one following Ag-nps administration as previously report by us [31], the present study was undertaken to investigate roles of mtUPR and mitophagy on the Ag-nps induced mitochondrial dysfunction.

\section{Materials and Methods}

\section{Materials}

Silver nanoparticles (The physical characteristics of the particles according to the manufacturers data are: size $(\leq 100 \mathrm{~nm})$, purity $(99.5 \%$ based on trace metals analysis), surface area $\left(5.0 \mathrm{~m}^{2} / \mathrm{g}\right)$, density 10.49 $\mathrm{g} / \mathrm{cm}^{3}$ (lit.), Polyvinylpyrrolidone (PVP) as dispersant [32]; the mean diameter of the dispersed particles was $22.32 \pm 7.07 \mathrm{~nm}$ (determined by TEM imaging) as previously described [31]) and anti-LC3B antibody were purchased from Sigma-Aldrich (St. Louis, MO, USA). Hsp70, Hsp60 and Hsp10 antibodies were supplied by Enzo Life Science (Farmingdale, NY, USA). Ubiquitin, mtHsp70 and C/EBP $\beta$ antibodies were obtained from Santa Cruz Biotechnology, Inc. (Delaware Avenue, California, USA). Mouse monoclonal antibody specific to CHOP, rabbit polyclonal antibody against BNIP3 and rabbit polyclonal antibody against voltage-dependent anion channel (VDAC) were products of Thermo Scientific (Rockford, Ill, USA), Cell Signaling Technology (Danvers, MA, USA) and NOVUS Biologicals (Littleton, CO, USA), respectively. ClpP and glyceraldehyde-3-phosphate dehydrogenase (GAPDH) antibodies were supplied by Abcam (Cambridge, MA, USA). ATP determination kit was obtained from Molecular Probes (Paisley, $\mathrm{UK})$. Other chemicals and reagents were of analytical grade.

\section{Animal model and administration of Ag-nps}

Male Sprague-Dawley rats ( BioLasco Taiwan Co., Ltd) weighing from 300 to $350 \mathrm{~g}$ were used in our study. All animal experiments in this study were housed and used in accordance with the approval of the Animal Care Committee of the Kaohsiung Medical University. Rats were selected randomly and divided into two groups (6-8 rats in each group): sham operated and treatment groups. Food and drinking water were supplied ad libitum. The experiments were carried out in the same manner as previously described by us [31]. Briefly, $150 \mathrm{mg}$ of Ag-nps powder was homogenously dispersed in $1.5 \mathrm{ml}$ of an aqueous solution. Under light ether anesthesia, Ag-treated rats received an intraperitoneally (i.p.) injection of Ag-nps suspension at a dose of $500 \mathrm{mg} \mathrm{kg}{ }^{-1}$. Sham-operated rats received equal volume of deionized water, and time-matched with Ag-treated group. Tissue samples were obtained for biochemical analysis at 6, 12, 18 and $24 \mathrm{hr}$ after treatment.

\section{Determination of ATP content}

Hepatic ATP content was determined by using a commercially available ATP determination kit (Molecular Probes) [31]. The assay system is based on production of light caused by the reaction of ATP

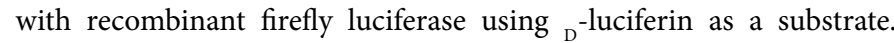
In brief, isolated liver proteins were added into a standard reaction solution containing firefly luciferase, ${ }_{\mathrm{D}}$-luciferin and DTT according to the manufacturer's instructions. ATP standard solutions were prepared using known concentrations of ATP from a concentration of $1000 \mu \mathrm{M}$ down to blank. Under the catalytic activity of luciferase, the luminescence evoked by the interaction of ATP and luciferin was recorded by a luminometer (HIDEX, Turku, Finland) and the ATP content was then calculated.

\section{Isolation of mitochondrial and cytosolic fractions from rat liver}

Specimens of liver were rapidly removed and immediately rinsed with ice-cold SEH buffer (0.25 M sucrose; 0.5 mM EGTA; 3 mM HEPES, $\mathrm{pH}$ 7.2). The livers were minced into small pieces, homogenized eight strokes by a Teflon pestle operated at 1500 r.p.m in ice-cold SEH buffer, and then centrifuged at $800 \mathrm{~g}$ for $10 \mathrm{~min}$ to remove unbroken cells and nuclei. The $800 \mathrm{~g}$ supernatant was centrifuged twice at $9500 \mathrm{~g}$ for $10 \mathrm{~min}$ to separate cytosol (supernatant) and mitochondrial (pellet) fractions. The resulting supernatant was stored at $-80^{\circ} \mathrm{C}$ and used as cytosolic fraction later. The resulting pellet was gently resuspended in ice-cold SEH buffer and recentrifuged at $9500 \mathrm{~g}$ for $10 \mathrm{~min}$ to remove any microsomal contamination. The final pellet was resuspended in ice-cold SEH buffer, stored at $-80^{\circ} \mathrm{C}$, and used as a mitochondrial 


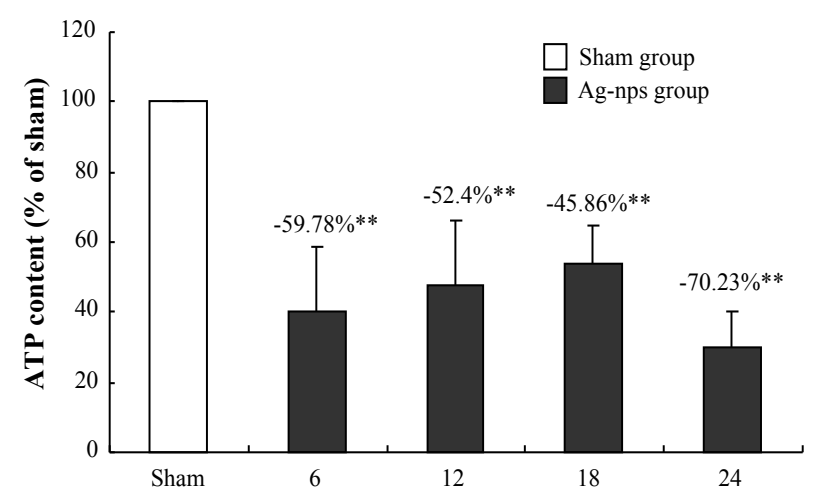

Time after treatment (hr)

Figure 1: Time-course of intracellular ATP content in rat liver tissues following Ag-nps administration. The cellular ATP contents in liver tissues were measured using a luciferase reaction-based assay kit as described under Material and Methods. Values were means \pm SD $(n=6-8)$. " $p<0.01$ compared to sham group.

fraction later. The entire procedure was performed at $4^{\circ} \mathrm{C}$. Protein concentrations were determined using the Bio-Rad protein assay (BioRad, Hercules, Calif), with bovine serum albumin as the standard.

\section{Preparation of nuclear extracts from rat liver}

Hepatic nuclear extracts were prepared by the method of Tran et al. [33] with modification. Fresh livers $(2 \mathrm{~g})$ were Dounce homogenized in two volumes of TKM buffer $(50 \mathrm{mM}$ Tris- $\mathrm{HCl}, \mathrm{pH} 7.5,25 \mathrm{mM}$ $\mathrm{KCl}, 5 \mathrm{mM} \mathrm{MgCl}$ ), $0.25 \mathrm{M}$ sucrose, $0.1 \%(\mathrm{v} / \mathrm{v}) 2$-mecaptoethanol (2-ME), followed by filtration through two layers of nylon gauze. The homogenates $(2 \mathrm{ml})$ were centrifugation at $2400 \mathrm{~g}$ for $10 \mathrm{~min}$, resuspended in $1 \mathrm{ml}$ TKM containing $0.25 \mathrm{M}$ sucrose, mixed with two volumes of TKM containing $2.3 \mathrm{M}$ sucrose, and layered on $1 \mathrm{ml}$ of TKM containing $2.3 \mathrm{M}$ sucrose. The mixtures were centrifuged at $82800 \mathrm{~g}$ for $1 \mathrm{hr}$ in an AT6 rotor (Hitachi CS120GXL, Tokyo, Japan). The resulting pellets were resuspended in TKM containing $0.25 \mathrm{M}$ sucrose and $0.5 \%$ $(\mathrm{v} / \mathrm{v})$ Triton X-100, and the suspensions were allowed to stand for 10 min to dissolve nuclear membranes. The suspensions were repelleted at $2000 \mathrm{~g}$ for $5 \mathrm{~min}$, and resuspended in buffer $\mathrm{A}(50 \mathrm{mM}$ Tris- $\mathrm{HCl}, \mathrm{pH} 7.5$, $10 \mathrm{mM}$ EDTA, $0.1 \%(\mathrm{v} / \mathrm{v})$ 2-ME, 1xprotease inhibitor cocktail (Roche, Penzberg, Germany)). After $10 \mathrm{~min}$ on ice, the suspensions were Dounce homogenized and centrifuged at $7800 \mathrm{~g}$ for $5 \mathrm{~min}$. The resulting supernatants were retained. The resulting pellets were rehomogenized in buffer B (50 mM Tris- $\mathrm{HCl}, \mathrm{pH} 7.5,0.42 \mathrm{M} \mathrm{NaCl}, 0.1 \mathrm{mM}$ EGTA, $5 \%$ (v/v) glycerol, $0.1 \%$ (v/v) 2-ME, 1xprotease inhibitor cocktail (Roche)), and recentrifugation at $7800 \mathrm{~g}$ for $10 \mathrm{~min}$. The final supernatants and the supernatants retained were pooled, stored at $-80^{\circ} \mathrm{C}$, and used as nuclear extracts in later days. The entire procedure was performed at $4^{\circ} \mathrm{C}$.

\section{Western blot analysis}

Hsp70, Hsp60, Hsp10, ClpP, CHOP, C/EBP $\beta$, BNIP3, LC3-II and Ub-proteins were quantified by the standard Western blot analysis [34]. Briefly, equal amounts of protein $(50 \mu \mathrm{g})$ extract were denatured, separated by $8 \%$ (for Hsp70, Hsp60, C/EBP $\beta$ ) or 12\% (for Hsp10, ClpP, CHOP, BNIP3, LC3-II and Ub-proteins) of sodium dodecyl sulfate (SDS)-polyacrylamide gel electrophoresis (PAGE) electrophoresis. The nonspecific binding sites were blocked by $5 \%$ non-fat milk in Trisbuffer saline (TBS) for $1 \mathrm{hr}$. Blots were immunoblotted with primary antibodies against Hsp70, Hsp60, Hsp10, ClpP, CHOP, C/EBP $\beta$, BNIP3,
LC3-II and Ub-proteins $(1: 1000)$ at $4^{\circ} \mathrm{C}$ overnight. After that, the membranes were washed and hybridized with horseradish peroxidaseconjugated species-specific secondary antibodies at room temperature for $1 \mathrm{hr}$. The VDAC and GAPDH were used as loading controls for mitochondrial and cytosolic fractions, respectively. Gels were stained with Coomassie blue to demonstrate the equal loading of ub-proteins, and the relative densities were normalized to the total amount of protein determined by Coomassie blue. Blots were developed using an enhanced chemiluminescence (ECL) detection reagent (Amersham Life Science), visualized on FUJI Medical X-ray film, and the relative densities were quantified.

\section{Statistical analysis}

All results were presented as mean \pm standard deviations. Statistical comparison of the data was performed using one-way analysis of variance (ANOVA) followed by Tukey's least significant difference procedure. A $P$-value of less than 0.05 was accepted as statistically significant.

\section{Results}

Figure 1 depicts time course of changes in ATP content of liver tissues following Ag-nps administration. Exposure to Ag-nps induced a rapid and marked reduction (-59.8\%) in ATP content $6 \mathrm{hr}$ post administration. The ATP content remained at the reduced levels $(-52.4 \%$ to $-70.2 \%)$ throughout the remaining experimental period (12-24 hr). These results demonstrate that mitochondrial energy metabolism was impaired by Ag-nps administration and the impairment seems to be irreversible.

Figure 2 shows changes in mitochondrial ubiquitinated proteins expression in liver tissues at various time points following i.p. injection of Ag-nps. As shown in Figure 2, mitochondrial Ub-protein expressions were increased in a time-dependent manner in Ag-nps administered group. The increases were statistically significant $(p<0.05): 50 \%$ at 12 $\mathrm{hr}, 63.7 \%$ at $18 \mathrm{hr}$, and $71.8 \%$ at $24 \mathrm{hr}$ following Ag-nps administration. These results demonstrate that Ag-nps caused mitochondrial proteins misfolding in the liver following Ag-nps administration.

Figure 3 depicts expression of Hsp70, Hsp60, Hsp10 and ClpP in rat liver following Ag-nps administration. As depicted in Figure 3, no upregulations of mitochondrial Hsp70, Hsp60 (Figure 3A and 3B) and ClpP (Figure 3D) expression were observed 6-24 hr following Ag-nps injection, although Ag-nps caused a significant increase in mitochondrial Hsp10 protein levels (Figure 3C). The cytosolic Hsp70 (Figure 3A) protein expression was gradually increased in a timedependent manner; while the cytosolic protein levels of Hsp60 (Figure 3B) and Hsp10 (Figure 3C) showed a transient increase at $12 \mathrm{hr}$ and 18 hr. These results indicate that Ag-nps failed to induce mtUPR-related chaperones and proteases.

Figure 4 shows time course of changes in nuclear $\mathrm{CHOP}$ and $\mathrm{C} /$ EBP $\beta$ expression in rats following intraperitoneal administration of Ag-nps. There were no changes in $\mathrm{CHOP}$ and $\mathrm{C} / \mathrm{EBP} \beta$ protein expressions in liver tissues throughout the entire experimental period. Putting together the results of Figure 3 and 4, it is apparent that the total mtUPR-related chaperone content and transcription factors were not appropriately induced during the period of unfolded protein accumulation under Ag-nps-induced mitochondrial stress.

Figure 5 depicts changes in LC3-II (Figure 5A) and BNIP3 protein expression (Figure 5B) in liver tissues at various time points following i.p. injection of Ag-nps. As shown in Fig. 5A, LC3-II protein expression was significantly increased at $6 \mathrm{hr}$ as compared to sham group $(p<0.01)$. 
A
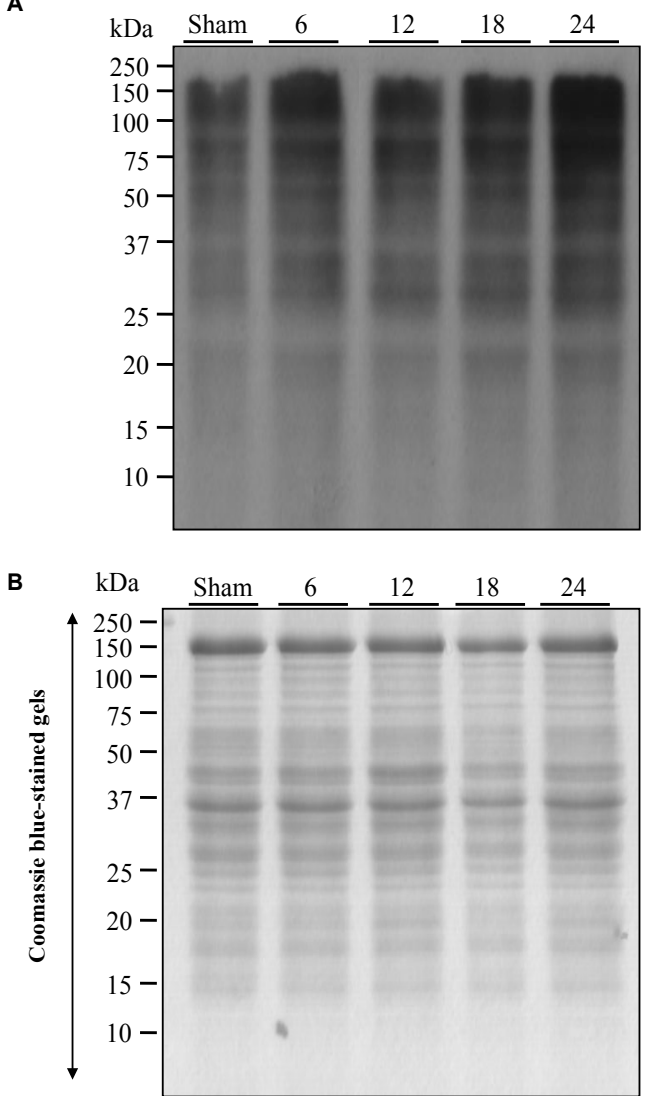

C

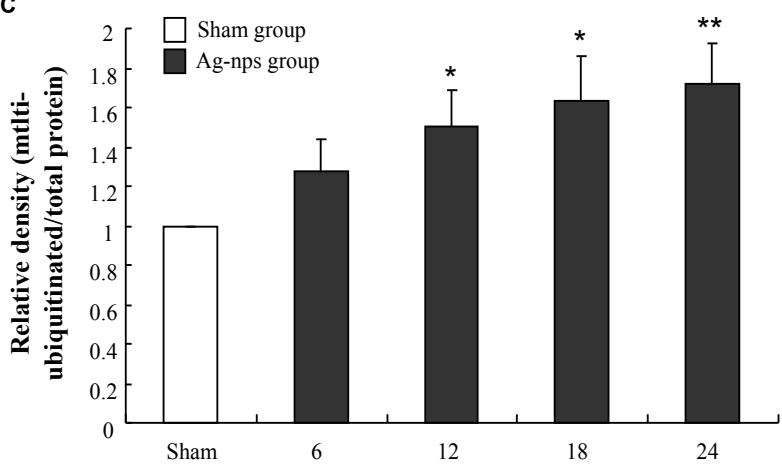

Time after treatment (hr)

Figure 2: Changes in mitochondrial ubiquitinated proteins expression in liver tissues at various time points following i.p. injection of Ag-nps. A: Mitochondrial ubiquitinated proteins expression based on Western blot analysis. B: Coomassie blue-stained gels demonstrating equal loading of the samples. C: The relative densities were normalized from part A to the total amount of protein measured from part $B$. The experiments were performed as described under Material and Methods. Empty columns represent sham groups while filled columns represent Ag-nps treatment groups. Molecular weight size markers $(\mathrm{kDa})$ are indicated at the left. Values were means $\pm \mathrm{SD}$ $(n=8),{ }^{*} p<0.05$ and ${ }^{* *} p<0.01$ compared to sham group.

The LC3-II protein expression was gradually declined thereafter in a time-dependent manner (12-24 hr). As shown in Figure 5B, the BNIP3 was increased at $6 \mathrm{hr}(p<0.05)$, peaked at $12 \mathrm{hr}(p<0.01)$, and remained elevated from $18 \mathrm{hr}(p<0.01)$ to $24 \mathrm{hr}(p<0.05)$ following Ag-nps administration. These findings suggest that the mitophagy was induced at early stage following Ag-nps administration and it was gradually diminished thereafter.

Figure 6 summarizes the interrelationships among changes in Ub-proteins, autophagy/mitophagy markers and ATP content in rat livers as a function of time following Ag-nps administration. As show in Figure 6, autophagy marker LC3-II was markedly induced at early stage which accompanied by a moderate increase in mitophagy marker BNIP3 and a progression and sustained upregulation of Ubproteins following Ag-nps administration. Meanwhile, ATP content in the liver was decreased at early stage and remained depressed during the entire experimental period. These results indicate that activation of autophagy/mitophagy markers, upregulation of Ub-proteins, and reduction of high energy phosphate compound co-existed in the liver following Ag-nps administration.

\section{Discussion}

Since the use of Ag-nps onto the surface of more consumer products, direct contact by human with Ag-nps is increasing, therefore; the concern about their potential toxicity is also growing. We have previously demonstrated that apoptosis was induced mainly through early reduction of intracellular ATP content in the liver after Ag-nps treatment [31]. This study is a continuation of our previous work to investigate role of in vivo mitochondrial stress response in coping with Ag-nps.

Proper folding of proteins is essential for normal cell function and survival. Disturbance of protein folding and deposition of aggregated proteins in the cells are pathologic to numerous protein misfolding diseases including neurodegenerative diseases (Alzheimer's and Parkinson's disease), phenylketonuria, alpha-1-antitrypsin deficiency, and short-chain acyl-CoA dehydrogenase deficiency [35-37]. For this reason, protein quality control system is important in maintaining protein folding homeostasis. In general, environmental factors such as temperature, ionic strength, $\mathrm{pH}$, and oxidation agents are widely accepted to having a triggering impact on the misfolding of proteins [38] This notion was supported by our present findings that mitochondrial Ub-proteins in the liver began to accumulate at $12 \mathrm{hr}$, and the increases were maintained up to $24 \mathrm{hr}$ following Ag-nps administration. There are two possibilities which could account for this event. First, the formation of silver-protein complexes due to strong binding of Ag-nps to thiol groups of the mitochondrial inner membrane proteins moieties, that in turn altered protein conformation and perturbed its function $[39,40]$. Alternatively, ROS produced by mitochondria after exposure to Ag-nps directly interact with mitochondrial proteins and eventually damaging their functions.

Cells have evolved complex systems to cope with diverse challenges that imposed on mitochondrial integrity. The first line of defence is provided by monitoring of mitochondrial protein quality control [41]. The mtUPR has two major mitochondrial chaperone systems to facilitate protein folding in the matrix: $\mathrm{mtHsp} 70$ and Hsp60/Hsp10 chaperone machineries. As nascent mitochondrial peptides emerge from the ribosome and translocated across membranes into the mitochondria, they are bound by the mtHsp70 via its peptide binding domain to prevent misfolding and aggregation. Some proteins require further folding assistance by the Hsp60/Hsp10 chaperonin complexes. Meanwhile, upexpression of the two transcription factors: namely $\mathrm{CHOP}$ and $\mathrm{C} / \mathrm{EBP} \beta$, is one feature that promotes unfolded protein response to mitochondrial stress. Since we found that molecular chaperones, protease and transcription factors in the mitochondrial fraction were not fittingly activated, even though the molecular chaperones in the 
Citation: Lee TY, Liu MS, Huang LJ, Lue SI, Tsai TZN, et al. (2014) The Immediate Mitochondrial Stress Response in Coping with Systemic Exposure of Silver Nanoparticles in Rat Liver. J Nanomed Nanotechnol 5: 220. doi: 10.4172/2157-7439.1000220

A

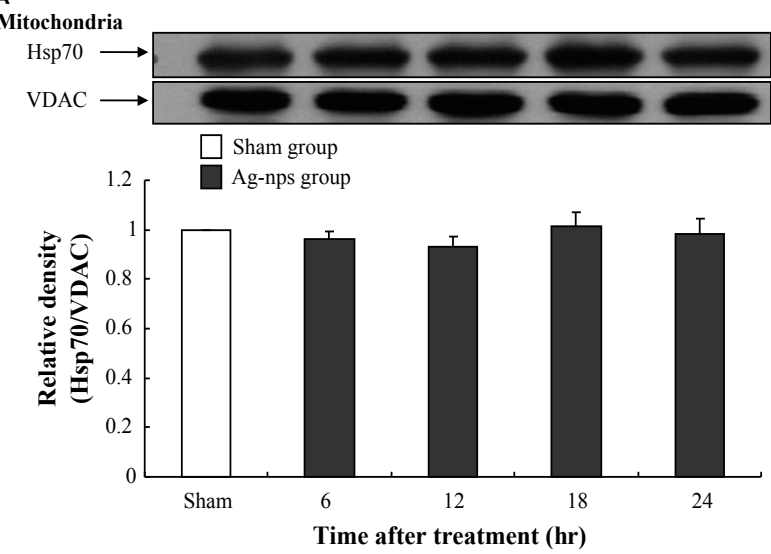

Cytosol
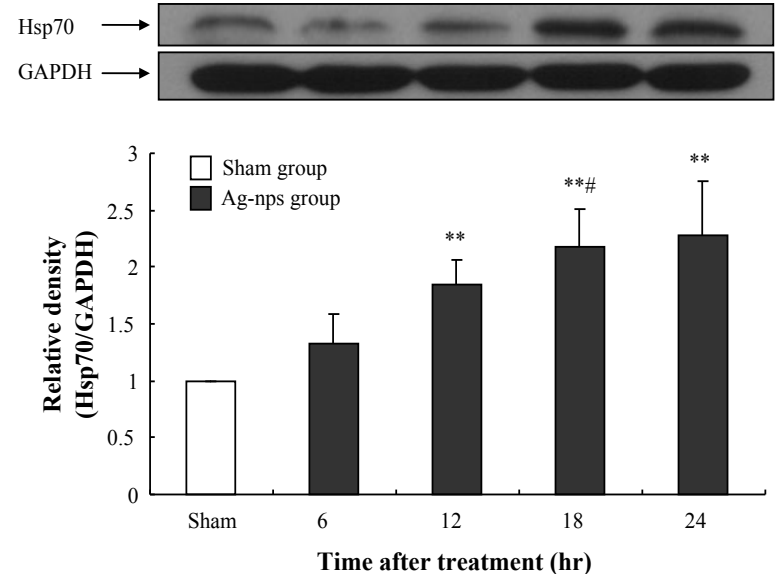

B

Mitochondria
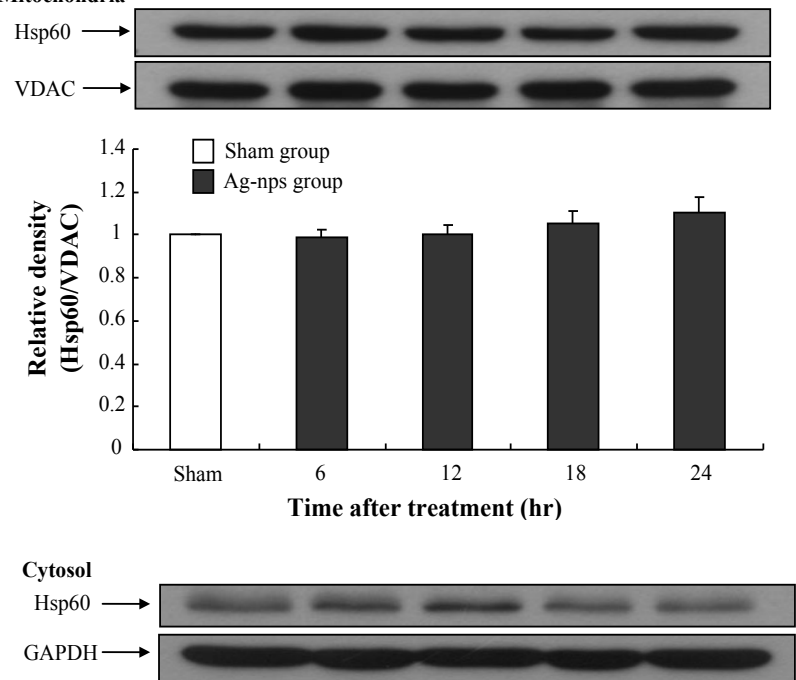

Mitochondria
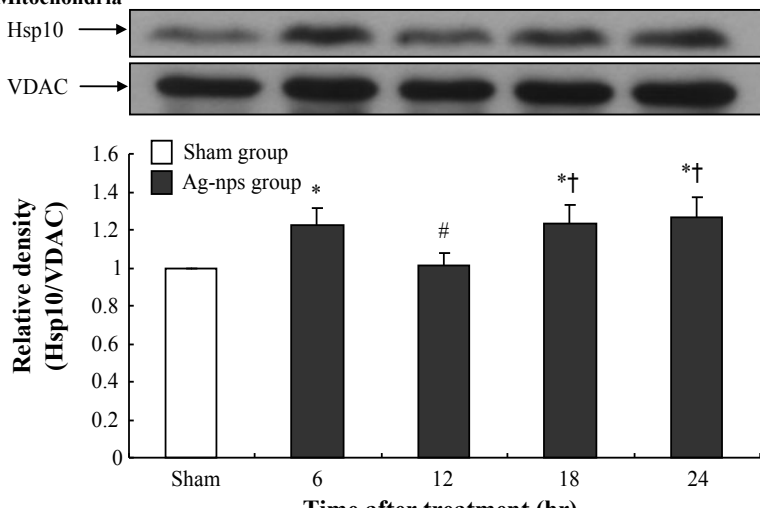

Time after treatment (hr)
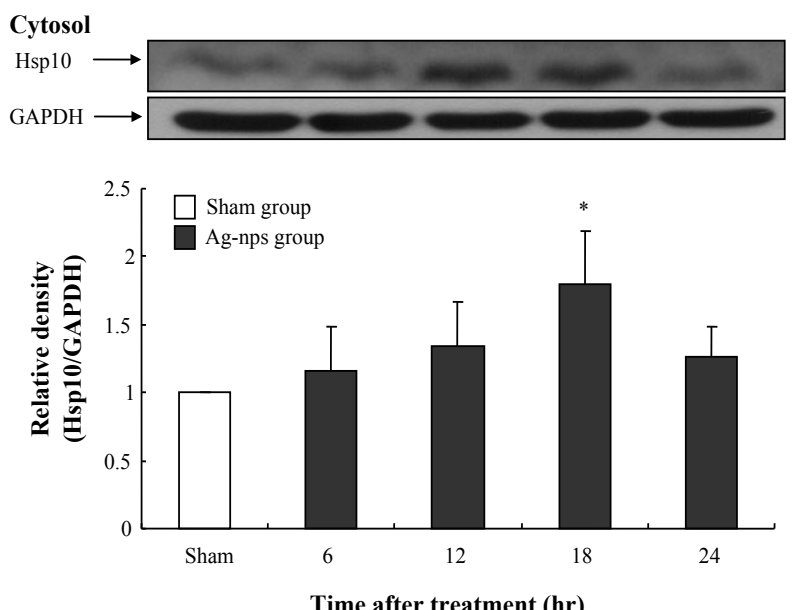

Time after treatment (hr)

Mitochondria
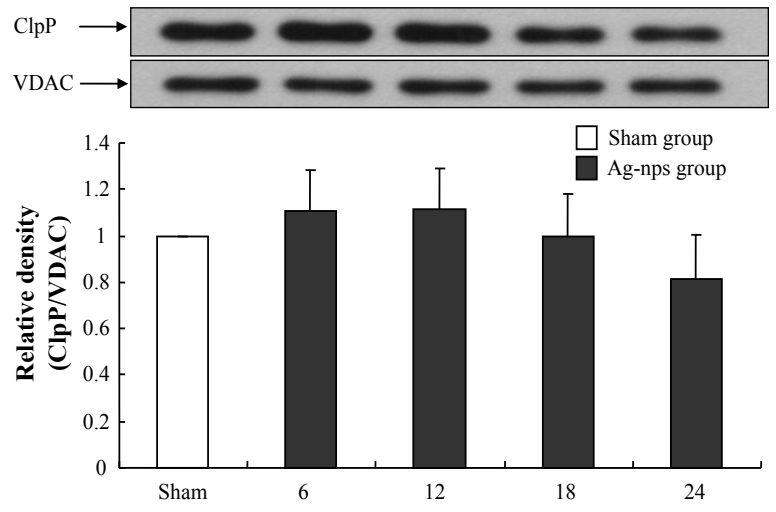

Time after treatment (hr)

$\square$ Sham group

Figure 3: Expression of Hsp70, Hsp60, Hsp10 and ClpP were observed in rat liver following Ag-nps administration. Upper panels show the representative immunoblots of molecular chaperones Hsp70 (A), Hsp60 (B), Hsp10 (C) and protease ClpP (D) in mitochondrial and cytosolic fractions while lower panels depict densitometric analysis of immunoblots. Experiments were carried out as described under Materials and Methods. The VDAC and GAPDH were used as mitochondria and cytosolic markers, respectively. Values were means \pm SD $(n=8)$. ${ }^{*} p<0.05$ and ${ }^{* *} p<0.01$ versus sham group; ${ }^{*} p<0.05$ versus 6 hr group; ${ }^{\dagger} p<0.05$ versus 12 hr group. 


\section{A}
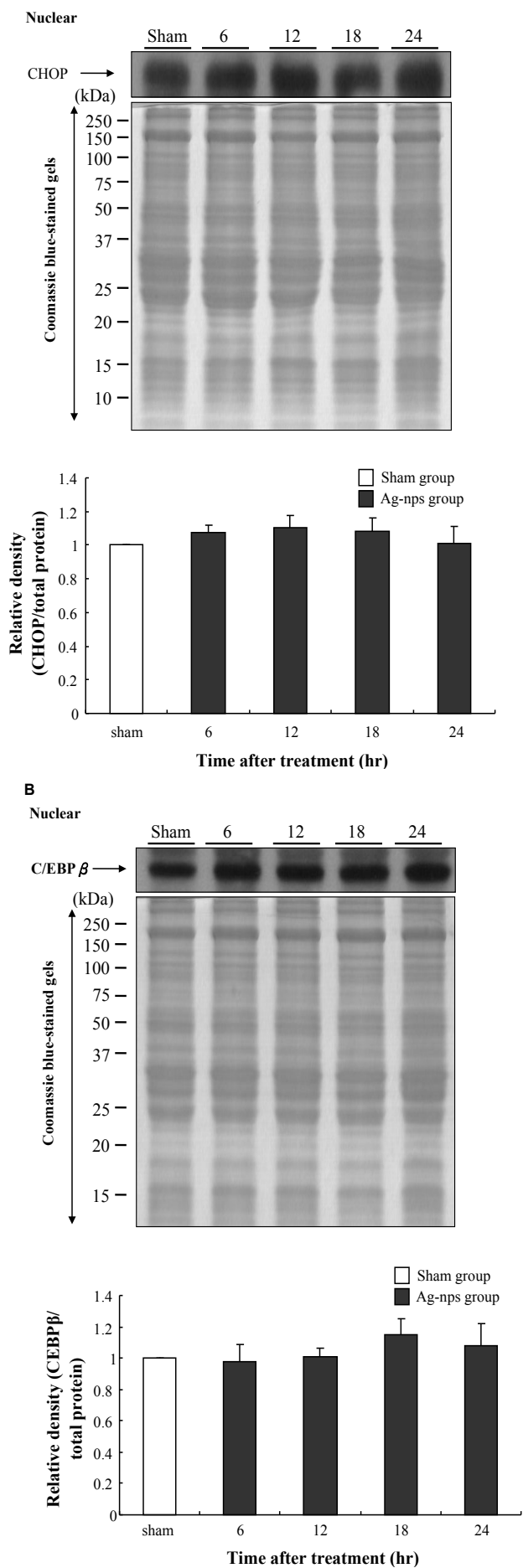

Figure 4: Time course of changes in nuclear CHOP (A) and C/EBP $\beta$ (B) expression in rats following intraperitoneal administration of Ag-nps. Upper panels of part A and part B: Nuclear CHOP and C/EBP $\beta$ proteins expression were analyzed by Western blot analysis. Middle panels of part $\mathrm{A}$ and part B: Coomassie blue-stained gels demonstrating equal loading of the samples. Molecular weight size markers $(\mathrm{kDa})$ are indicated at the left of each image. Lower panels of part $A$ and part $B$ : The relative densities were normalized from upper panel to the total amount of protein measured from middle panel. Nuclear protein fractions were processed for Western blotting as described under Materials and Methods. Values were means $\pm \operatorname{SD}(n=7)$. cytosolic fraction were transiently activated, it is unlikely that mtUPR is a significant defensive factor attempting to ameliorate mitochondrial stress following Ag-nps administration.

Protein quality control works at multiple levels: monitoring, processing, and elimination of mitochondrial misfolded/unfolded proteins by molecular chaperones and proteases. If the first line of defense such as mtUPR fails, a second line of defence such as mitophagy comes into play by timely elimination of dysfunctional mitochondria to protect cells from harming disordered mitochondrial metabolism. Several studies have reported that BNIP3 is a pro-apoptotic BH3only protein which is associated with mitochondrial dysfunction and cell death $[28,42,43]$. BNIP3 functions as a receptor for the selective clearance of mitochondria in cells (mitophagy) and this process is independent of its role in apoptotic signaling [44]. Recently, it was reported that BNIP3 can directly interact with autophagy protein LC3-II (microtubule-associated protein light chain 3), suggesting that binding of BNIP3 to LC3-II on the autophagosome tethers the mitochondrion to the autophagosome $[28,45,46]$. In this study, we

A
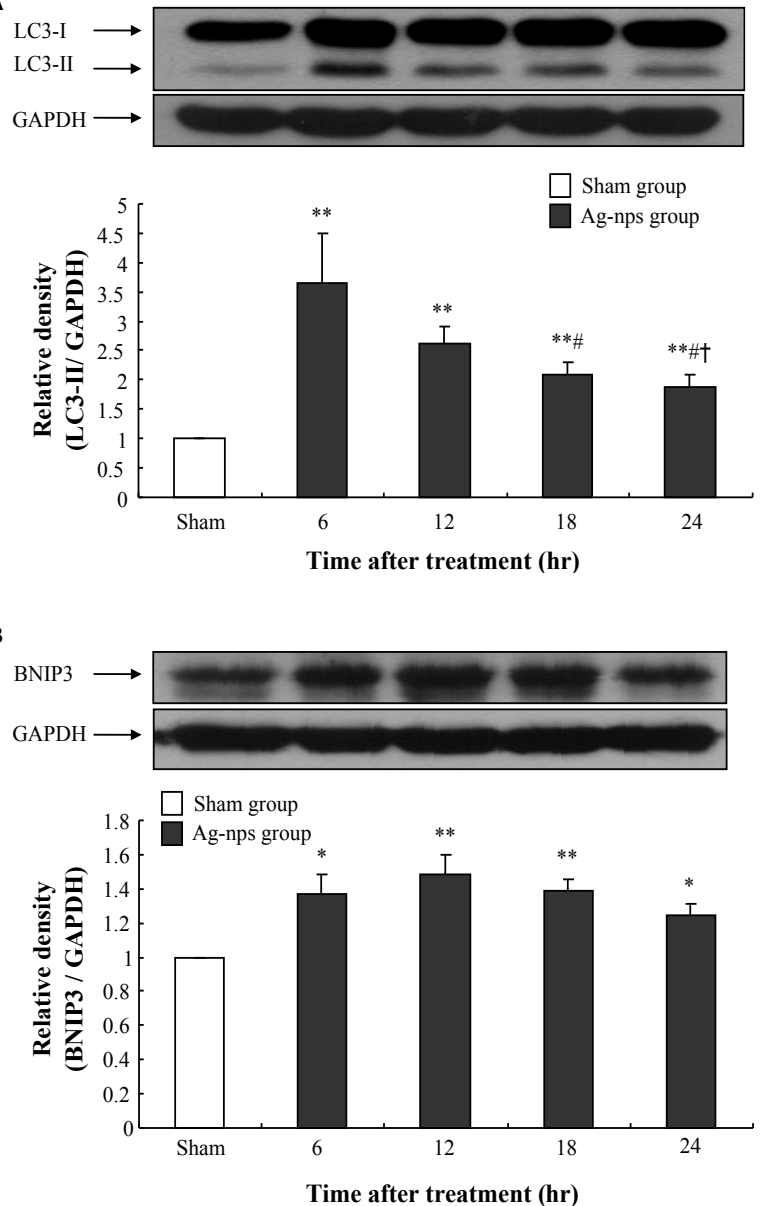

Figure 5: Changes in LC3-II (autophagy marker) and BNIP3 (mitophagy marker) protein expression in rat liver at different time points after $i . p$. injection of Ag-nps. Upper images show the representative immunoblots of autophagy marker LC3-II (A) and mitophagy marker BNIP3 (B) in liver tissues while lower images depict densitometric analyses of immunoblots. GAPDH gene was used as an internal loading control. Values were means $\pm \operatorname{SD}(n=8)$, " $p<0.05$ and ${ }^{* *} p<0.01$ versus sham group; ${ }^{*} p<0.05$ versus $6 \mathrm{hr}$ group; ${ }^{\dagger} p<0.05$ versus 12 hr group. 


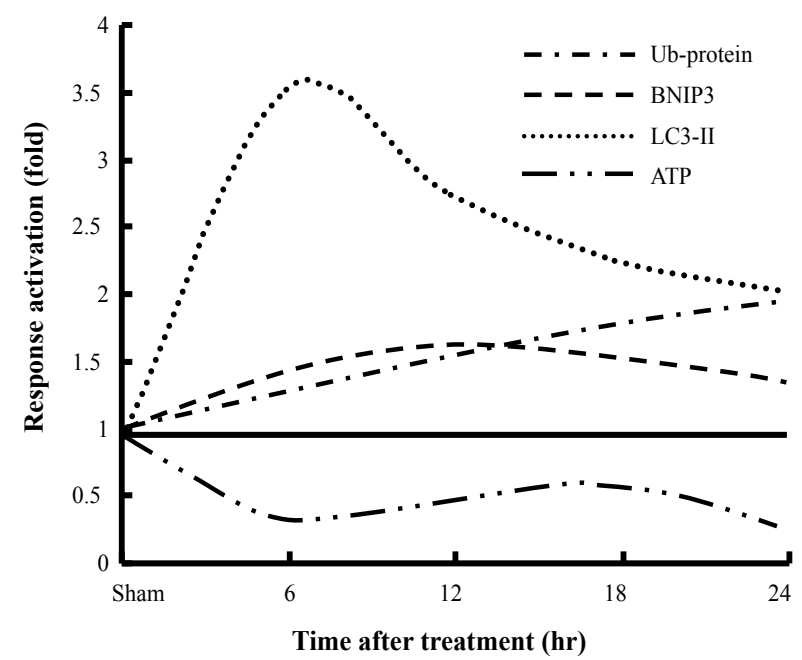

Figure 6: Interrelationships among changes in Ub-proteins, autophagy/ mitophagy markers and ATP content in rat livers as a function of time following Ag-nps administration. Data in Figures 1, 2 and 5 were used and replotted as a function of time following Ag-nps administration.

found that LC3-II and BNIP3 protein expressions in hepatocytes were increased at $6 \mathrm{hr}$ post Ag-nps administration, and the levels remained increased during the $24 \mathrm{hr}$ experimental period. These findings demonstrate that autophagy/mitophagy was activated shortly after Ag-nps administration. The activation of mitophagy would protect mitochondrial function from deterioration, however; ATP contents were found to decrease following Ag-nps administration indicating that the protective effects of mitophagy on mitochondrial function is likely compromised by others factors such as upregulation of Ub-proteins or dysfunction of fission and fusion of mitochondrial membranes [41]. Further studies are needed to verify this possibility.

Studies of acute toxicity and biodistribution of silver nanoparticles have provided evidence of interaction between mitochondria protein/ DNA and Ag-nps in a variety of mammalian cell systems. In human lung fibroblast and glioblastoma cells treated with Ag-nps. Asha Rani et al. [47] found that the nanoparticles deposit inside the mitochondria and other organelles such as nucleus. Deposition of Ag-nps in mitochondria eventually altered normal function of mitochondria through disruption of electron transport chain, increase in ROS generation, and decrease in ATP yield. In addition, some of the Ag-nps in the cell nucleus interacted with DNA, leading to cell cycle arrest in the G2/M phase [47]. In primary mouse dermal fibroblasts and liver cells treated with silver nanoparticles, Arora et al. [48] found the presence of silver nanoparticles inside the mitochondria and cytoplasm of treated cells. The presence of silver nanoparticles inside the mitochondria eventually caused oxidative stress [48]. In mouse embryo fibroblast cell line (NIH 3T3) treated with silver nanoparticles, it has been found that cytochrome c was released from mitochondria into the cytosol $24 \mathrm{hr}$ after treatment, whereas cytochrome $\mathrm{c}$ remained in the mitochondria in untreated cells [49]. The cytochrome $c$ released from mitochondria was associated with disruption of mitochondrial membrane potential, resulting from interaction between mitochondrial membrane proteins and Ag-nps [50].

In summary, activation of autophagy/mitophagy markers coexisted with upregulation of Ub-proteins and reduction of ATP content without a change in mtUPR in the liver following Ag-nps administration.
The fact that ATP content was reduced at early stage and remained depressed during the entire experimental period following Ag-nps administration, indicating that the protective effects of autophagy/ mitophagy markers on the control of mitochondrial function were overwhelmed by the detrimental activities of Ub-proteins, and the counter-balance of the two systems eventually resulting in an impaired mitochondrial function, i.e., reduction of ATP content.

\section{Acknowledgement}

This work was supported by grants from National Science Council (NSC 1022320-B-037-023).

\section{References}

1. Ahamed M, Alsalhi MS, Siddiqui MK (2010) Silver nanoparticle applications and human health. Clin Chim Acta 411: 1841-1848.

2. Chaloupka K, Malam Y, Seifalian AM (2010) Nanosilver as a new generation of nanoproduct in biomedical applications. Trends Biotechnol 28: 580-588.

3. Chen X, Schluesener HJ (2008) Nanosilver: a nanoproduct in medical application. Toxicol Lett 176: 1-12.

4. Quadros ME, Marr LC (2010) Environmental and human health risks of aerosolized silver nanoparticles. J Air Waste Manag Assoc 60: 770-781.

5. Xue Y, Zhang S, Huang Y, Zhang T, Liu X, et al. (2012) Acute toxic effects and gender-related biokinetics of silver nanoparticles following an intravenous injection in mice. J Appl Toxicol 32: 890-899.

6. Tiwari DK, Jin T, Behari J (2011) Dose-dependent in-vivo toxicity assessment of silver nanoparticle in Wistar rats. Toxicol Mech Methods 21: 13-24.

7. Kim YS, Kim JS, Cho HS, Rha DS, Kim JM, et al. (2008) Twenty-eight-day oral toxicity, genotoxicity, and gender-related tissue distribution of silver nanoparticles in Sprague-Dawley rats. Inhal Toxicol 20: 575-583.

8. Li Y Bhalli JA, Ding W, Yan J, Pearce MG, et al. (2014) Cytotoxicity and genotoxicity assessment of silver nanoparticles in mouse. Nanotoxicology 8: 36-45.

9. Raimundo N (2014) Mitochondrial pathology: stress signals from the energy factory. Trends Mol Med 20: 282-292.

10. Doris KS, Rumsby EL, Morgan BA (2012) Oxidative stress responses involve oxidation of a conserved ubiquitin pathway enzyme. Mol Cell Biol 32: 44724481.

11. Papa L, Germain D (2014) SirT3 regulates the mitochondrial unfolded protein response. Mol Cell Biol 34: 699-710.

12. Finkel T (2011) Signal transduction by reactive oxygen species. J Cell Biol 194 7-15.

13. Miki H, Funato $Y$ (2012) Regulation of intracellular signalling through cysteine oxidation by reactive oxygen species. J Biochem 151: 255-261.

14. Jovaisaite V, Mouchiroud L, Auwerx J (2014) The mitochondrial unfolded protein response, a conserved stress response pathway with implications in health and disease. J Exp Biol 217: 137-143.

15. Altieri DC (2011) Mitochondrial compartmentalized protein folding and tumor cell survival. Oncotarget 2: 347-351.

16. Costa CS, Ronconi JV, Daufenbach JF, Gonçalves CL, Rezin GT, et al. (2010) In vitro effects of silver nanoparticles on the mitochondrial respiratory chain. Mol Cell Biochem 342: 51-56.

17. Pratsinis A, Hervella P, Leroux JC, Pratsinis SE, Sotiriou GA (2013) Toxicity of silver nanoparticles in macrophages. Small 9: 2576-2584.

18. Teodoro JS, Simões AM, Duarte FV, Rolo AP, Murdoch RC, et al. (2011) Assessment of the toxicity of silver nanoparticles in vitro: a mitochondrial perspective. Toxicol In Vitro 25: 664-670.

19. Bressan E, Ferroni L, Gardin C, Rigo C, Stocchero M, et al. (2013) Silver nanoparticles and mitochondrial interaction. Int J Dent 2013: 312747.

20. Barbour JA, Turner N (2014) Mitochondrial stress signaling promotes cellular adaptations. Int J Cell Biol 2014: 156020.

21. Haynes CM, Fiorese CJ, Lin YF (2013) Evaluating and responding to mitochondrial dysfunction: the mitochondrial unfolded-protein response and beyond. Trends Cell Biol 23: 311-318. 
Citation: Lee TY, Liu MS, Huang LJ, Lue SI, Tsai TZN, et al. (2014) The Immediate Mitochondrial Stress Response in Coping with Systemic Exposure of Silver Nanoparticles in Rat Liver. J Nanomed Nanotechnol 5: 220. doi: 10.4172/2157-7439.1000220

Page 8 of 8

22. Ryan MT, Hoogenraad NJ (2007) Mitochondrial-nuclear communications. Annu Rev Biochem 76: 701-722.

23. Pellegrino MW, Nargund AM, Haynes CM (2013) Signaling the mitochondria unfolded protein response. Biochim Biophys Acta 1833: 410-416.

24. Baker MJ, Tatsuta T, Langer T (2011) Quality control of mitochondrial proteostasis. Cold Spring Harb Perspect Biol 3.

25. Kotiadis VN, Duchen MR Osellame LD2 (2014) Mitochondrial quality control and communications with the nucleus are important in maintaining mitochondria function and cell health. Biochim Biophys Acta 1840: 1254-1265.

26. Knaevelsrud $H$, Simonsen A (2010) Fighting disease by selective autophagy of aggregate-prone proteins. FEBS Lett 584: 2635-2645.

27. Youle RJ, Narendra DP (2011) Mechanisms of mitophagy. Nat Rev Mol Cell Biol 12: 9-14.

28. Hanna RA, Quinsay MN, Orogo AM, Giang K, Rikka S, et al. (2012) Microtubule-associated protein 1 light chain 3 (LC3) interacts with Bnip3 protein to selectively remove endoplasmic reticulum and mitochondria via autophagy. $J$ Biol Chem 287: 19094-19104.

29. Lee J, Giordano S, Zhang J (2012) Autophagy, mitochondria and oxidative stress: cross-talk and redox signalling. Biochem J 441: 523-540.

30. Frank M, Duvezin-Caubet S, Koob S, Occhipinti A, Jagasia R, et al. (2012) Mitophagy is triggered by mild oxidative stress in a mitochondrial fission dependent manner. Biochim Biophys Acta 1823: 2297-2310.

31. Lee TY, Liu MS, Huang LJ, Lue SI, Lin LC, et al. (2013) Bioenergetic failure correlates with autophagy and apoptosis in rat liver following silver nanoparticle intraperitoneal administration. Part Fibre Toxicol 10: 40

32. Chen D, Xi T, Bai J (2007) Biological effects induced by nanosilver particles: in vivo study. Biomed Mater 2: S126-128.

33. Tran HT, Ulke A, Morrice N, Johannes CJ, Moorhead GB (2004) Proteomic characterization of protein phosphatase complexes of the mammalian nucleus. Mol Cell Proteomics 3: 257-265.

34. Huang LJ, Dong HP, Chuang IC, Liu MS, Yang RC (2012) Attenuation of mitochondrial unfolded protein response is associated with hepatic dysfunction in septic rats. Shock 38: 642-648.

35. Desideri E, Martins LM (2012) Mitochondrial Stress Signalling: HTRA2 and Parkinson's Disease. Int J Cell Biol 2012: 607929.

36. Bernales S, Soto MM, McCullagh E (2012) Unfolded protein stress in the endoplasmic reticulum and mitochondria: a role in neurodegeneration. Front Aging Neurosci 4: 5 .
37. Gregersen N, Bross P, Vang S, Christensen JH (2006) Protein misfolding and human disease. Annu Rev Genomics Hum Genet 7: 103-124.

38. Herczenik E, Gebbink MF (2008) Molecular and cellular aspects of protein misfolding and disease. FASEB J 22: 2115-2133.

39. Drake PL, Hazelwood KJ (2005) Exposure-related health effects of silver and silver compounds: a review. Ann Occup Hyg 49: 575-585

40. Almofti MR, Ichikawa T, Yamashita K, Terada H, Shinohara Y (2003) Silver ion induces a cyclosporine a-insensitive permeability transition in rat liver mitochondria and release of apoptogenic cytochrome C. J Biochem 134: 43-49.

41. Tatsuta T, Langer $T$ (2008) Quality control of mitochondria: protection against neurodegeneration and ageing. EMBO J 27: 306-314.

42. Quinsay MN, Thomas RL, Lee Y, Gustafsson AB (2010) Bnip3-mediated mitochondrial autophagy is independent of the mitochondrial permeability transition pore. Autophagy 6: 855-862.

43. Gustafsson AB (2011) Bnip3 as a dual regulator of mitochondrial turnover and cell death in the myocardium. Pediatr Cardiol 32: 267-274.

44. Thomas RL, Kubli DA, Gustafsson AB (2011) Bnip3-mediated defects in oxidative phosphorylation promote mitophagy. Autophagy 7: 775-777.

45. Kubli DA, Gustafsson ÅB (2012) Mitochondria and mitophagy: the yin and yang of cell death control. Circ Res 111: 1208-1221.

46. Rikka S, Quinsay MN, Thomas RL, Kubli DA, Zhang X, et al. (2011) Bnip3 impairs mitochondrial bioenergetics and stimulates mitochondrial turnover. Cell Death Differ 18: 721-731.

47. AshaRani PV, Low Kah Mun G, Hande MP, Valiyaveettil S (2009) Cytotoxicity and genotoxicity of silver nanoparticles in human cells. ACS Nano 3: 279-290.

48. Arora S, Jain J, Rajwade JM, Paknikar KM (2009) Interactions of silver nanoparticles with primary mouse fibroblasts and liver cells. Toxicol Appl Pharmacol 236: 310-318.

49. Hsin YH, Chen CF, Huang S, Shih TS, Lai PS, et al. (2008) The apoptotic effect of nanosilver is mediated by a ROS- and JNK-dependent mechanism involving the mitochondrial pathway in NIH3T3 cells. Toxicol Lett 179: 130-139.

50. Piao MJ, Kang KA, Lee IK, Kim HS, Kim S, et al. (2011) Silver nanoparticles induce oxidative cell damage in human liver cells through inhibition of reduced glutathione and induction of mitochondria-involved apoptosis. Toxicol Lett 201 $92-100$ 